\title{
Advances in the research on lymphangiogenesis in carcinoma tissues (Review)
}

\author{
XIAOFEI QIU ${ }^{1 *}$, SHUJUAN YAO ${ }^{1 *}$ and SHIQIAN ZHANG ${ }^{2}$ \\ ${ }^{1}$ Department of Gynaecology and Obstetrics, The Centre People's Hospital of Tengzhou, Tengzhou 277500; \\ ${ }^{2}$ The State-Key Discipline of Obstetrics and Gynaecology, Department of Gynaecology and Obstetrics, \\ Qilu Hospital of Shandong University, Ji'nan 250012, P.R. China
}

Received March 30, 2010; Accepted May 12, 2010

DOI: 10.3892/ol_00000102

\begin{abstract}
Metastatic spread of tumors is an important prognostic factor for cancer patients. The effect of angiogenesis on cancer cell proliferation and metastatic spread has been confirmed. However, less attention has been focused on research involving tumor lymphangiogenesis as opposed to research on tumor angiogenesis, due to the lack of specific markers for lymphatic vessel endothelial cells (LVECs). Recently, the improvement of isolation techniques for LVECs and the discovery of specific LVEC markers such as vascular endothelial growth factor receptor-3 (VEGFR-3), podoplanin, lymphatic vessel endothelial hyaluronan receptor-1 (LYVE-1) and Prox1 have led to advances in research involving lymphangiogenesis in carcinoma tissues. New lymphatic vessels in tumor tissues may originate from bone marrow endothelial progenitor cells, directly from the preexisting lymphatic vessels, and even by cell transformation. Peritumoral lymphatic vessels play a more important role in the process of tumor metastasis by providing more channels for lymphatic invasion and metastatic spread. The molecular mechanism of tumor lymphangiogenesis is complicated, and numerous factors such as VEGF-A, platelet-derived growth factors (PDGFs), hepatocyte growth factor (HGF), fibroblast growth factor-2 (FGF-2), and angiopoietins (Ang) are directly or indirectly involved in the process. However, it has been demonstrated that the VEGF-C/VEGF-D/VEGFR-3 signaling pathways are the most important mechanism underlying tumor lymphangiogenesis.
\end{abstract}

Correspondence to: Dr Shiqian Zhang, The State-Key Discipline of Obstetrics and Gynecology, Department of Gynaecology and Obstetrics, Qilu Hospital of Shandong University, Ji'nan, Shandong 250012, P.R. China

E-mail: zhangshiqian370112@126.com

*Contributed equally

Key words: carcinoma, lymphangiogenesis, lymphatic metastasis, lymphatic vessel density

\section{Contents}

1. Introduction

2. LVEC markers

3. Mechanism of lymphangiogenesis in carcinoma tissues

4. Morphology of tumor lymphatic vessels and their correlation with clinical pathology

5. Molecular mechanism of tumor lymphangiogenesis

6. Conclusions

\section{Introduction}

Lymphatic vessels play an important role, not only in physiological processes such as environmental stability, immunologic surveillance and fat absorption, but also in pathological processes such as lymphedema, tissue repair, inflammatory reaction, and particularly, in the metastatic spread of tumors. Metastatic spread of tumors is an important prognostic factor for cancer patients. The effect of angiogenesis on cancer cell proliferation and metastatic spread has been confirmed. However, less attention has been focused on research involving tumor lymphangiogenesis versus research on tumor angiogenesis, owing to the lack of specific markers for lymphatic vessel endothelial cells (LVECs). Recently, improvement in the isolation techniques of LVECs and the discovery of specific LVEC markers have led to advances in research involving lymphangiogenesis in carcinoma tissues. This review evaluates the advances in research regarding lymphangiogenesis in carcinoma tissues.

\section{LVEC markers}

Vascular endothelial growth factor receptor-3 (VEGFR-3), also known as Flt-4, is the earliest and most widely used LVEC marker. It is specifically expressed on the lumen of LVECs in normal adult tissues. However, the liver, splenic blood sinuses, tissues involved in wound repair and neovascular endothelial cells of carcinomas are also known to express VEGFR-3. Thus, VEGFR-3 as an LVEC marker does not have a high specificity.

Podoplanin is an LVEC marker with a relatively high specificity and is mainly expressed in micro-LVECs. Podoplanin 
is an integrated cytoplasm/cell membrane mucoprotein that was initially confirmed in the glomerular podocyte. However, its biological function has yet to be determined. Schacht et al (1) reported that podoplanin plays an important role in the regulation of the process of lymphangiogenesis. Knock-out of the Podoplanin gene in mice leads to a reduction in the transport capacity of lymphatic vessels, an expansion of lymphatic vessels and congenital lymphedema. Studies have demonstrated that podoplanin is expressed in squamous cell carcinoma and in some germ cell tumors, suggesting a possible association of podoplanin with tumor progression (2).

Lymphatic vessel endothelial hyaluronan receptor-1 (LYVE-1), which is homologous with CD44, is expressed on the surface of LVECs and is considered to be the most valuable marker of LVECs. It is assumed that LYVE-1 is associated with the metabolism of hyaluronic acid in the extracellular matrix (3).

Prox 1 is a homeobox gene transcriptional factor and is specifically expressed in embryos, adult normal tissues, and intratumoral LVECs. One study revealed that Prox1 is involved in the formation of the LVEC phenotype during lymphatic vascular development, indicating that it can be used as a marker of LVECs (4).

However, the aforementioned markers are not entirely specific for LVECs. Therefore, a certain amount of crossreaction between LVECs and microvascular endothelial cells was observed during the staining procedure. The immunohistochemical method of double dyeing is used to label lymphatic vessels in order to discriminate between blood and lymphatic vessels. In addition, a recent gene analysis of lymphatic and blood vascular endothelial cells revealed certain novel LVECspecific genes, such as macrophage mannose receptor-1, integrin- $\alpha 9$ and chemotactic factor CCL20.

\section{Mechanism of lymphangiogenesis in carcinoma tissues}

The mechanism of tumor lymphangiogenesis is complicated and has yet to be determined. New lymphatic vessels in tumor tissues may originate from bone marrow endothelial progenitor cells, directly from preexisting lymphatic vessels, and even by cell transformation.

Bone marrow endothelial progenitor cells express CD34 and VEGFR-2 and are a cellular subgroup that originates from endothelial cells lining the circulatory system. These endothelial progenitor cells proliferate, permeate into, and fuse with vascular endothelial cells and are involved in tumor angiogenesis under pathological conditions. Salven et al (4) reported that besides CD34 and VEGFR-2, certain endothelial progenitor cells also express the LVEC marker VEGFR-3, indicating that these cells may be the progenitor cells of LVECs. Moreover, the endothelial progenitor cells are involved in tumor lymphangiogenesis in the same manner as tumor angiogenesis mentioned above.

However, it has been shown that new intratumoral lymphatic vessels originate by a 'budding' of preexisting lymphatic vessels. He et al (5) found that new lymphatic vessels mainly originated from preexisting lymphatic vessels of tissues undergoing lymphangiogenesis in carcinoma cases, but no or few vessels originated from bone marrow endothelial progenitor cells.
Other investigators showed that body macrophages, not only promote the proliferation of preexisting LVECs, but also migrate into the tissue stroma and are directly transformed into LVEC clusters to communicate with preexisting lymphatic vessels (6). This finding suggests that some cells are transformed into LVECs to generate new lymphatic vessels. However, whether tumor cells generate new lymphatic vessels in this manner has yet to be confirmed.

\section{Morphology of tumor lymphatic vessels and their correlation with clinical pathology}

In the process of tumor lymphangiogenesis, lymphangiogenic factors, such as VEGF-C and VEGF-D, combine with the specific receptor VEGFR-3, which is distributed in LVECs, and promote LVEC proliferation to generate new lymphatic vessels. These new intratumoral lymphatic vessels have structures that are similar to new physiological lymphatic vessels, but the former have thin vessel walls and lack tight junctions, which are beneficial for the promotion of tumor metastasis (7).

Notably, whether such new intratumoral lymphatic vessels exist and play a role in tumor metastasis has yet to be determined. Animal trials have shown that new lymphatic vessels are generated by activating lymphangiogenic factors and are associated with lymph node metastasis of tumors (8). However, no similar result has been reported in humans. Human intratumoral lymphatic vessels consist of only 2 or 3 LVECs, which are small, irregular and collapsed. Consequently, these vessels are thought to provide no activities, although some of them show positive staining for proliferating cell nuclear antigens (9). A possible explanation for this finding is the rapid proliferation and tumor tissue division in human cancer cells, which increases the interstitial hydrostatic pressure and blocks the lumen of new lymphatic vessels (10). In addition, tumor invasion can disrupt lymphatic vessels (11). Although the intratumoral lymphatic vessels may not provide any activity, the increase in lymphatic vessels can increase the risk of lymph node metastasis of tumor cells, and subsequently increase the intratumoral lymphatic vascular density (ILVD) associated with lymph node metastasis and poor prognosis of cancer patients. Maula et al investigated LYVE-1-positive lymphatic vessels in 97 patients with head and neck squamous cell carcinoma. Results of these authors showed that ILVD was also associated with a risk of local recurrence in patients (12). Sipos et al investigated pancreatic endocrine cancers, which were characterized by vascular invasion and metastasis. In their study, it was shown that the levels of ILVD increased in certain types of tumors (13). This suggests that intratumoral lymphatic vessels are not only associated with lymph node metastasis, local recurrence and poor prognosis, but may also be associated with the malignant progression of tumors. Therefore, ILVD can be used to predict risk of lymph node metastasis and prognosis of cancer patients.

Peritumoral lymphatic vessels are believed to play a more important role in the process of tumor metastasis by providing more channels for lymphatic invasion and metastatic spread $(14,15)$. Using a dye-adsorption test, Padera et al (14) confirmed that functional intratumoral lymphatic vessels were mostly located in the peritumoral zones in that the peritumoral 
lymphatic vessels had an expanded lumen that was disordered and distorted and had visible cancer emboli in their lumen, compared with the collapsed and flat intertumoral lymphatic vessels. However, Straume et al (15) found that an increase in peritumoral lymphatic vessels density (PLVD) in the cutaneous melanoma was often accompanied by lymphocyte invasion and was positively correlated with the prognosis and survival of patients. One possible explanation for this finding is that increased peritumoral lymphatic vessels promote the recruitment of antigen-presenting cells (APCs), thereby stimulating immune response against the tumors.

\section{Molecular mechanism of tumor lymphangiogenesis}

The molecular mechanism of tumor lymphangiogenesis is complicated, and numerous factors are directly or indirectly involved in this process. Currently, the VEGF-C/VEGF-D/ VEGFR-3 signaling pathways are believed to be the most important mechanism underlying tumor lymphangiogenesis (16-22).

$V E G F-C$ and VEGF-D. VEGF-C or VEGF-D combines with the tyrosine kinase (TK) receptor VEGFR-3 expressed in LVECs, thereby promoting LVEC proliferation and lymphangiogenesis via a series of intracellular signaling pathways. Clinical trials on human tumors indicated that the expression of VEGF-C or VEGF-D is associated with lymph node metastasis and the poor prognosis of patients with cancer (16). Using relevant animal models, it was confirmed that VEGF-C or VEGF-D plays an important role in the regulation of tumor lymphangiogenesis and lymph node metastasis in that the overexpression of VEGF-C was accompanied by intra- and/or peri-LVD and lymphatic metastasis (17). However, Karkkainen et al (18) reported that $\mathrm{Vegfc}^{-/-}$mice cannot generate lymphatic vessels via budding, leading to the dysplasia of lymphatic vessels in tissues and prenatal death from excessive fluid accumulation in tissues. VEGF-C or VEGF-D administration can compensate this defect in Vegfc $^{-/}$mice. Haiko et al investigated a mutant mouse model in which the mutation site was located downstream of the $V E G F R-3$ gene. The results revealed that dysgenopathy of lymphatic vessels in $\mathrm{Vegfc}^{+/-}$mice was ameliorated after Kemurinein 14 promoters induced the overexpression of the human or mouse $V E G F-D$ gene in the mice (19). Therefore, the VEGF-C/VEGF-D/VEGFR-3 signaling pathways play an important role in the regulation of the tumor lymphangiogenesis process. This finding provides a potential target for gene therapy against lymphangiogenesis in cancers.

Stacker et al investigated a mouse model injected with 293EBNA cancer cells which do not express VEGF-C or VEGF-D (20). VEGF-D facilitated tumor lymphangiogenesis, while the monoclonal antibody against VEGF-D blocked this effect. Lin et al (21) investigated a nude murine model that was injected with renal carcinoma Caki-2 cells and reported that Caki-2 cells promoted tumor lymphangiogenesis. The tumors were transfected with soluble VEGFR-3 (sVEGFR3-Fc) vectors via the mediation of adenovirus after being xenografted. The results indicated that $\mathrm{SVEGFR} 3-\mathrm{Fc}$ vector transfection led to the suppression of lumen expansion, an increase in the number of peritumoral lymphatic vessels, and a reduction in lymph node metastasis. Makinen et al (22) investigated cutaneous lymphangiogenesis in a transgenic mouse model and revealed that Kemurinein 14 promoters enhanced the overexpression of VEGF-C to promote the development of cutaneous lymphatic hyperplasia. However, the transfection of soluble VEGFR-3 led to the competitive inhibition of VEGF-C, owing to the specific combination between VEGFR-3 and VEGF-C, thus leading to the development of cutaneous alymphoplasia in mice.

In addition, VEGF-C interferes with the process of tumor lymphatic metastasis in various ways, e.g., by changing LVEC adhesion and promoting the expression of chemotactic factors and their receptors.

$V E G F-A$. The main function of VEGF-A is to promote angiogenesis. Its receptor is VEGFR-2 which is expressed by some LVECs. Clinical (23) and animal trials (24) have shown that the expression of VEGF-A in cancers promotes tumor lymphangiogenesis and is positively correlated with lymph node metastasis. VEGF-A may facilitate tumor lymphangiogenesis in a direct or an indirect manner, i.e., directly by stimulating VEGFR-2 or indirectly by activating the VEGF-C/VEGF-D/ VEGFR-3 signaling pathways. However, whether VEGF-A acts directly or indirectly has yet to be determined.

Platelet-derived growth factors. Members of the plateletderived growth factor (PDGF) families comprise PDGF-AA, PDGF-AB, PDGF-BB, PDGF-CC and PDGF-DD. PDGFR- $\alpha$ and PDGFR- $\beta$ are receptors with TK enzyme activity in that the activation of PDGFR- $\alpha$ and PDGFR- $\beta$ can facilitate tumor cell proliferation and angiogenesis. It has been determined that PDGF-BB may also be a lymphatic vessel growth factor and may facilitate tumor lymphangiogenesis, independent of the VEGF-C/VEGF-D/VEGFR-3 signaling pathways (25). However, this effect needs to be verified by conducting more clinical trials and animal tests.

Other growth factors. The main function of the hepatocyte growth factor (HGF) is to regulate liver cell proliferation as well as to stimulate proliferation and migration of some epithelial cells. HGF plays various roles in tissue repair and tumor invasion. Recent research has shown that HGF may facilitate lymphangiogenesis in a direct or an indirect manner, i.e., directly by activating the HGF receptor (HGF-R, also known as MET/c-met) and indirectly by activating the VEGF-C/VEGF-D/VEGFR-3 signaling pathways (26).

Insulin-like growth factors (IGFs) include IGF-1 and IGF-2 and their receptors IGF-1R and IGF-2R. Björndahl et al (27) found that IGF-1 and IGF-2 promoted LVEC proliferation and migration in vitro. IGF-1 and IGF-2 also facilitated corneal lymphangiogenesis in mice in vivo, and this effect of lymphangiogic facilitation mediated by IGF-1 was independent of the VEGF-C/VEGF-D/VEGFR-3 signaling pathways.

Fibroblast growth factor-2 (FGF-2) is another factor that may facilitate lymphangiogenesis. In a mouse corneal model, FGF-2 facilitated lymphangiogenesis, and the effect was indirectly elicited by the activation of the VEGF-C/VEGF-D/ VEGFR-3 signaling pathways (28).

Angiopoietins. Angiopoietins comprise Ang-1 and Ang-2, and Tie2 is their receptor. Ang-1 positively regulates vascular 
remodeling and stability, but Ang-2 has an antagonistic effect. Previous studies showed that Ang-1 is able to facilitate the growth of lymphatic vessels via 'budding', and this effect can be suppressed by transfection of the soluble protein VEGFR-3, indicating that Ang-1 acts via the VEGF-C/VEGF-D/VEGFR-3 signaling pathways (29). Although no reports are currently available on the Ang-2-mediated facilitation of lymphangiogenesis, clinical trials in gastric cancer indicated that Ang- 2 is related to tumor lymph node metastasis (30).

\section{Conclusions}

Although current research on tumor lymphangiogenesis is incomplete and a number of conflicting issues need to be resolved, we believe that suppression of tumor lymphangiogenesis and lymphatic metastasis provide novel ways of treating cancers. The treatment modalities use gene therapy based on advances in the research on tumor lymphangiogenesis and elucidate its underlying mechanism.

\section{Acknowledgements}

The study was supported by Shandong Natural Science Foundation (Y2008C70).

\section{References}

1. Schacht V, Ramirez MI, Hong YK, et al: T1alpha/podoplanin deficiency disrupts normal lymphatic vasculature formation and causes lymphedema. EMBO J 22: 3546-3556, 2003.

2. Schacht V, Dadras SS, Johnson LA, Jackson DG, Hong YK and Detmar M: Up-regulation of the lymphatic marker podoplanin, a mucin-type transmembrane glycoprotein, in human squamous cell carcinomas and germ cell tumors. Am J Pathol 166: 913-921, 2005.

3. Wigle JT, Harvey N, Detmar M, et al: An essential role for Prox 1 in the induction of the lymphatic endothelial cell phenotype. EMBO J 21: 1505-1513, 2002.

4. Salven P, Mustjoki S, Alitalo R, Alitalo K and Rafii S: VEGFR-3 and CD133 identify a population of CD $34^{+}$lymphatic/vascular endothelial precursor cells. Blood 101: 168-172, 2003.

5. He Y, Rajantie I, Ilmonen M, et al: Preexisting lymphatic endothelium but not endothelial progenitor cells are essential for tumor lymphangiogenesis and lymphatic metastasis. Cancer Res 64: 3737-3740, 2004.

6. Kerjaschki D: The crucial role of macrophages in lymphangiogenesis. J Clin Invest 115: 2316-2319, 2005.

7. Ji RC and Kato S: Lymphatic network and lymphangiogenesis in the gastric wall. J Histochem Cytochem 51: 331-338, 2003.

8. Mattila MM, Ruohola JK, Karpanen T, Jackson DG, Alitalo K and Harkonen PL: VEGF-C induced lymphangiogenesis is associated with lymph node metastasis in orthotopic MCF-7 tumors. Int J Cancer 98: 946-951, 2002.

9. Franchi A, Massi D, Santucci M, et al: Inducible nitric oxide synthase activity correlates with lymphangiogenesis and vascular endothelial growth factor-C expression in head and neck squamous cell carcinoma. J Pathol 208: 439-445, 2006.

10. Koike C, McKee TD, Pluen A, et al: Solid stress facilitates spheroid formation: potential involvement of hyaluronan. Br J Cancer 86: 947-953, 2002.
11. Kato S, Shimoda H, Ji RC and Miura M: Lymphangiogenesis and expression of specific molecules as lymphatic endothelial cell markers. Anat Sci Int 81: 71-83, 2006.

12. Maula SM, Luukkaa M, Grenman R, Jackson D, Jalkanen S and Ristamaki R: Intratumoral lymphatics are essential for the metastatic spread and prognosis in squamous cell carcinomas of the head and neck region. Cancer Res 63: 1920-1926, 2003.

13. Sipos B, Klapper W, Kruse ML, Kalthoff H, Kerjaschki D and Kloppel G: Expression of lymphangiogenic factors and evidence of intratumoral lymphangiogenesis in pancreatic endocrine tumors. Am J Pathol 165: 1187-1197, 2004.

14. Padera TP, Kadambi A, di Tomaso E, et al: Lymphatic metastasis in the absence of functional intratumor lymphatics. Science 296: 1883-1886, 2002.

15. Straume O, Jackson DG and Akslen LA: Independent prognostic impact of lymphatic vessel density and presence of low-grade lymphangiogenesis in cutaneous melanoma. Clin Cancer Res 9: 250-256, 2003.

16. Saintigny P, Kambouchner M, Ly M, et al: Vascular endothelial growth factor-C and its receptor VEGFR-3 in non-small-cell lung cancer: concurrent expression in cancer cells from primary tumour and metastatic lymph node. Lung Cancer 58: 205-213, 2007.

17. Brakenhielm E, Burton JB, Johnson M, et al: Modulating metastasis by a lymphangiogenic switch in prostate cancer. Int $J$ Cancer 121: 2153-2161, 2007.

18. Karkkainen MJ, Haiko P, Sainio K, et al: Vascular endothelial growth factor $\mathrm{C}$ is required for sprouting of the first lymphatic vessels from embryonic veins. Nat Immunol 5: 74-80, 2004.

19. Haiko P, Makinen T, Keskitalo S, et al: Deletion of vascular endothelial growth factor C (VEGF-C) and VEGF-D is not equivalent to VEGF receptor 3 deletion in mouse embryos. Mol Cell Biol 28: 4843-4850, 2008.

20. Stacker SA, Caesar C, Baldwin ME, et al: VEGF-D promotes the metastatic spread of tumor cells via the lymphatics. Nat Med 7: 186-191, 2001.

21. Lin J, Lalani AS, Harding TC, et al: Inhibition of lymphogenous metastasis using adeno-associated virus-mediated gene transfer of a soluble VEGFR-3 decoy receptor. Cancer Res 65: 6901-6909, 2005.

22. Makinen T, Jussila L, Veikkola $\mathrm{T}$, et al: Inhibition of lymphangiogenesis with resulting lymphedema in transgenic mice expressing soluble VEGF receptor-3. Nat Med 7: 199-205, 2001.

23. Saad RS, Kordunsky L, Liu YL, Denning KL, Kandil HA and Silverman JF: Lymphatic microvessel density as prognostic marker in colorectal cancer. Mod Pathol 19: 1317-1323, 2006

24. Whitehurst B, Flister MJ, Bagaitkar J, et al: Anti-VEGF-A therapy reduces lymphatic vessel density and expression of VEGFR-3 in an orthotopic breast tumor model. Int J Cancer 121: 2181-2191, 2007.

25. Cao R, Bjorndahl MA, Religa P, et al: PDGF-BB induces intratumoral lymphangiogenesis and promotes lymphatic metastasis. Cancer Cell 6: 333-345, 2004.

26. Cao R, Bjorndahl MA, Gallego MI, et al: Hepatocyte growth factor is a lymphangiogenic factor with an indirect mechanism of action. Blood 107: 3531-3536, 2006.

27. Björndahl M, Cao R, Nissen LJ, et al: Insulin-like growth factors 1 and 2 induce lymphangiogenesis in vivo. Proc Natl Acad Sci USA 102: 15593-15598, 2005.

28. Chang LK, Garcia-Cardena G, Farnebo F, et al: Dose-dependent response of FGF-2 for lymphangiogenesis. Proc Natl Acad Sci USA 101: 11658-11663, 2004.

29. Morisada T, Oike Y, Yamada Y, et al: Angiopoietin-1 promotes LYVE-1-positive lymphatic vessel formation. Blood 105: 4649-4656, 2005.

30. Wang J, Wu K, Zhang D, et al: Expressions and clinical significances of angiopoietin-1, -2 and Tie2 in human gastric cancer. Biochem Biophys Res Commun 337: 386-393, 2005. 